\title{
De Ritis ratio (AST/ALT) as an independent predictor of poor outcome in patients with acute ischemic stroke
}

This article was published in the following Dove Press journal:

Neuropsychiatric Disease and Treatment

I5 June 2017

Number of times this article has been viewed

\author{
Fan Gao' \\ Chen Chen ${ }^{2}$ \\ Jun Lu' \\ Jie Zheng' \\ Xian-Cang $\mathrm{Ma}^{1,3}$ \\ Xing-Yun Yuan ${ }^{2}$ \\ Kang Huo ${ }^{2}$ \\ Jian-Feng $\mathrm{Han}^{2}$ \\ 'Clinical Research Center, \\ ${ }^{2}$ Department of Neurology, \\ ${ }^{3}$ Department of Psychiatry, The First \\ Affiliated Hospital of Xi'an Jiaotong \\ University, Xi'an, Shaanxi, China
}

Correspondence: Jian-Feng Han

Department of Neurology, The First

Affiliated Hospital of Xi'an Jiaotong

University, 277 Yanta West Road, Xi'an

71006I, Shaanxi, China

Tel +8629853236I4

Email hanjianfeng_xjtu@।63.com
Purpose: The aspartate transaminase/alanine transaminase ratio (De Ritis ratio, AAR) was reported to be associated with patients' prognosis in certain diseases recently. The objective of the current study was to determine the association between the AAR at admission and poor outcome at 3 months in acute ischemic stroke (AIS) patients.

Patients and methods: This retrospective cohort study included patients who experienced their first-ever AIS between June 2015 and March 2016. The primary outcome measure was a poor outcome at 3 months (modified Rankin Scale score $>2$ ). Multivariate logistic regression models were used to assess the relationship between AAR quartiles and clinical outcomes among the AIS patients. Receiver operating characteristic curve analysis was applied to identify the optimal cutoff for AAR in predicting the prognosis of AIS.

Results: In terms of the relationship between poor outcome and AAR, the adjusted odds ratio comparing the highest and lowest AAR quartiles was 2.15 (95\% confidence interval $=1.14-4.05$ ). An AAR of 1.53 was identified as the optimal cutoff. In a prespecified subgroup analysis according to the time from symptom onset to treatment ( $>24$ vs $\leq 24$ hours), there was no significant difference in the effect of AAR $>1.53$ between the two groups.

Conclusion: An increased AAR at admission is significantly associated with a poor outcome at 3 months in AIS patients.

Keywords: De Ritis ratio, stroke, prognosis, aspartate transaminase, alanine transaminase, modified Rankin scale, poor outcome

\section{Introduction}

Stroke is a severe disease with high disability and mortality rates and ranks as the second most common cause of death and the third most common cause of disability worldwide. ${ }^{1}$ Early prediction of the prognostic outcome of stroke patients is beneficial for ensuring that they receive the appropriate attention from clinicians when they are admitted to hospital, which may have positive effects on treatment and improve their functional recovery. Additional research on the prognosis of stroke is urgently needed.

Aspartate transaminase (AST) and alanine transaminase (ALT) are two blood enzymes that are reportedly positively correlated with functional outcome after acute ischemic stroke (AIS). ${ }^{2,3}$ This relationship might be due to the metabolic effects of AST and ALT in reducing the blood glutamate level. The release of excessive glutamate into the brain parenchyma after ischemic stroke would lead to neuronal injury. Lowering of the blood glutamate level according to the AST and ALT levels could therefore result in the transfer of glutamate from the brain into the peripheral blood (following the concentration gradient) and thereby exert a neuroprotective effect in AIS 
patients. ${ }^{4-8}$ Previous studies have also described AST as being associated with the cerebral infarct size and hemorrhagic transformation after AIS. ${ }^{9}, 10$ As a routine noninvasive laboratory test, the AST/ALT ratio (De Ritis ratio, AAR) represents the simultaneous alteration of AST and ALT levels, which has been used to assess the risk of critical limb ischemia in peripheral arterial occlusive disease and nonalcoholic fatty liver disease, and treated as a prognostic marker for diseases such as nonmetastatic renal cell carcinoma and upper urinary tract urothelial carcinoma. ${ }^{11-16}$

To the best of our knowledge, no previous study has considered using AAR to predict the prognosis of AIS or has integrated this ratio in a risk model of AIS. The modified Rankin Scale (mRS) is a commonly used index for evaluating the level of functional independence in the daily activities of stroke patients. It has been used in most literature focusing on the prognosis of stroke. Therefore, the present study aimed to, 1) determine the potential associations of AAR with the prognosis of AIS defined as mRS score at 3 months, and 2) explore if AAR is correlated with disease severity at admission indicated by the National Institutes of Health Stroke Scale (NIHSS), and bleeding during hospitalization.

\section{Materials and methods Patients}

This retrospective cohort study involved AIS patients who were consecutively admitted to the Department of Neurology, the First Affiliated Hospital of Xi' an Jiaotong University from June 2015 to March 2016. All of the patients were diagnosed in accordance with the World Health Organization criteria and diagnosis was further confirmed by brain computed tomography or magnetic resonance imaging in the hospital. Only patients with a first episode of ischemic stroke within 7 days from stroke onset were enrolled in the study. The following exclusion criteria were applied: 1) severe hepatic diseases, renal disease, or hematologic diseases; 2) previous disability; 3) cancer; 4) no AST and ALT data available at admission; or 5) no information on the functional outcome at 3 months after stroke. The study was approved by the ethics committee for medical research at the First Affiliated Hospital of Xi'an Jiaotong University. Because of the retrospective nature of the study, the requirement of written informed consent was waived by the review board. Patients' privacy information has been deleted for the whole process of data analysis.

\section{Data collection}

The following data on basic characteristics were collected: 1) demographic data - age and gender; 2) vascular risk factors - current smoking, current alcohol intake, hypertension, diabetes mellitus (DM), and dyslipidemia; 3) parameters measured at admission - systolic and diastolic blood pressures, Glasgow Coma Scale (GCS) score, symptom onset to treatment time (OTT); 4) blood serum levels in laboratory tests - fasting glucose, total cholesterol (TC), triglycerides (TGs), high-density lipoprotein cholesterol (HDL-C), lowdensity lipoprotein cholesterol (LDL-C), AST, and ALT; $5)$ recombinant tissue plasminogen activator (rtPA) treatment; and 6) stroke subtype.

The primary outcome was $\mathrm{mRS}$ score assessed at 3 months after stroke, with scores of 0-2 and 3-6 defined as good and poor outcomes, respectively., ${ }^{2,1718}$ Secondary outcomes were stroke severity at admission according to NIHSS and bleeding in any body part after treatment during hospitalization. The NIHSS score was dichotomized into $\leq 5$ and $>5 .{ }^{19}$ Both the mRS and NIHSS scores were evaluated by trained neurologists. mRS data after discharge were obtained by telephone.

Blood samples were collected from all AIS patients upon their arrival at hospital. Biochemical analysis was performed in the Clinical Laboratory of the First Affiliated Hospital of Xi'an Jiaotong University. All laboratory indicators were measured using an automatic biochemistry analyzer (BJ-G188; Hitachi, Tokyo, Japan).

Hypertension was defined as the current use of antihypertensive medications, a systolic blood pressure of $\geq 140 \mathrm{mmHg}$, and/or a diastolic blood pressure of $\geq 90 \mathrm{mmHg} .{ }^{20} \mathrm{DM}$ was defined as a previous diagnosis of and treatment for DM, a fasting glucose of $\geq 7.0 \mathrm{mmol} / \mathrm{L}$ ( $\geq 126 \mathrm{mg} / \mathrm{dL}$ ), a value of glucose concentration in the 2-hour oral glucose tolerance test, or a random plasma glucose concentration of $\geq 11.1 \mathrm{mmol} / \mathrm{L}$ $(200 \mathrm{mg} / \mathrm{dL})$ in the presence of the classic symptoms of hyperglycemia or a hyperglycemic crisis. ${ }^{21}$ Dyslipidemia was defined as $\mathrm{TC} \geq 6.22 \mathrm{mmol} / \mathrm{L}, \mathrm{TGs} \geq 2.26 \mathrm{mmol} / \mathrm{L}$, LDL-C $\geq 4.14 \mathrm{mmol} / \mathrm{L}$, or HDL-C $<1.04 \mathrm{mmol} / \mathrm{L} .{ }^{17}$ Stroke subtype was defined according to the TOAST (Trial of ORG 10172 in Acute Stroke Treatment) classification. ${ }^{22}$

\section{Statistical analysis}

The patients were categorized into four subgroups according to AAR quartiles. Basic characteristics information was compared between the four AAR groups using the KruskalWallis test for continuous variables and the Pearson $\chi^{2}$ test (modified by Fisher's exact test) for categorical variables. The AST and ALT levels and AAR were compared between two groups ( $m R S$ score $>2$ and $\leq 2$ ) with the Mann-Whitney $U$ test.

Univariate and multivariate nonconditional logistic regression models were used to assess the relationship 
between AAR quartiles and outcomes among the AIS patients. In a multivariate model, odds ratio (OR) and 95\% confidence interval $(95 \% \mathrm{CI})$ values in the AAR subgroups were determined after adjusting for sex, age, current smoking, alcohol intake, hypertension, DM, dyslipidemia, OTT, GCS score, and thrombolytic therapy with rtPA. The lowest quartile was treated as the reference.

Receiver operating characteristics (ROC) curve analysis was performed to identify the optimal cutoff for the continuous AAR to predict a poor outcome at 3 months after stroke. The optimal cutoff was determined based on maximizing the Youden index. The area under the ROC curve (AUC) with $95 \%$ CI, sensitivity, and specificity are presented. AAR was transformed into a dichotomous variable according to its optimal cutoff. This dichotomous variable of AAR was tested in a multivariate logistic regression model.

Subgroup analysis was conducted and stratified by the prespecified factor (OTT $\leq 24$ and $>24$ hours). Adjusted covariates included in the multivariate logistic regression model were sex, age, current smoking, alcohol intake, hypertension, DM, hyperlipidemia, OTT, GCS score, and thrombolytic therapy with rtPA. An interaction term was included in the multivariate logistic regression model to test if the effect of AAR changed with the OTT. A two-sided test with $P<0.05$ was considered to be statistically significant. All analyses were conducted using Statistical Package for the Social Sciences (SPSS) software version 24.0 (IBM, Chicago, IL, USA).

\section{Results}

In total, 421 AIS patients were finally included in the study. Patients who had serious hepatitis $(n=2)$, had no data on AST or ALT at admission ( $\mathrm{n}=23$ ), or were lost to follow-up at 3 months $(n=2)$ were excluded.

Basic information and clinical outcomes in the four subgroups are presented in Table 1. The AAR values were $<0.84,0.84-1.1,1.1-1.45$, and $>1.45$ in subgroups 1 , 2,3 , and 4 , respectively. The proportion of males was $75.5 \%$ in subgroup 1 and $50.5 \%$ in subgroup 4 . The proportion of males decreased as AAR increased $(P<0.001)$. The median age increased with increasing $\operatorname{AAR}(P<0.001)$. For vascular risk factors, the prevalence rates of smoking and alcohol intake were significantly lower when AAR was higher (both $P<0.05)$. The prevalence rates of hypertension, DM, and dyslipidemia did not vary significantly with AAR. The diastolic blood pressure, TGs, and ALT decreased, while HDL-C and AST increased (all $P<0.05$ ) with increasing AAR. The proportion of patients with a lower GCS score (0-7) was considerably higher in subgroup 4 than in the other three AAR subgroups $(P=0.005)$. The distribution of stroke subtypes according to TOAST classification varied widely between the four AAR subgroups $(P=0.007)$. With increasing AAR, the number of patients with the large-artery subtype of atherosclerosis appeared to decrease, while the number of cardioembolic stroke patients increased. The proportion of those receiving rtPA did not differ significantly between any of the AAR subgroups. In terms of clinical outcome, the percentages of patients with a poor outcome at 3 months were $34.9 \%, 44.8 \%, 43 \%$, and $58.3 \%$ in AAR subgroups $1-4$, respectively $(P=0.008)$. There were no evident differences in NIHSS score or bleeding during hospitalization among the AAR subgroups.

The AST and ALT levels and AAR are compared between good and poor outcomes in Figure 1. Patients with a poor outcome had a higher AAR (median =1.16), lower AST level $($ median $=18.1 \mathrm{IU} / \mathrm{L})$, and lower ALT level $($ median $=15.7 \mathrm{IU} / \mathrm{L})$ compared with patients with a good outcome $(1.06,20.1$, and 18.7 IU/L, respectively).

Independent associations between AAR and clinical outcomes were analyzed using multivariate logistic regression models (Table 2). Regarding the relationship between AAR and the primary outcome, the OR (95\% CI) values for the highest versus lowest AAR quartiles were 2.50 (1.36-4.60) for Model 1. After adjustment in Model 2 (Model 1 plus the GCS score), the independent relationship between AAR and poor outcome remained, but it was attenuated $(\mathrm{OR}=2.15$ and $95 \%$ CI $=1.14-4.05$ ). No independent association between AAR and other outcomes was detected.

ROC curve analysis detected that AAR $>1.53$ could be used to predict a poor outcome at 3 months poststroke with a sensitivity of $29.5 \%$ and a specificity of $85.3 \%$. The AUC was 0.580 (95\% CI $=0.525-0.635)$, as shown in Figure 2 . The effect of the optimal AAR cutoff $(>1.53)$ was evaluated in the multivariate logistic regression model (Table 3 ). In the total population, AAR $>1.53$ seemed to be independently associated with a poor outcome at 3 months ( $O R=1.89,95 \%$ $\mathrm{CI}=1.11-3.22, P=0.02)$. A prespecified subgroup analysis revealed that 198 patients were admitted to hospital within 24 hours of stroke onset, while in 223 patients this was delayed beyond 24 hours. AAR $>1.53$ increased the risk of a poor outcome at 3 months when OTT was longer than 24 hours $(\mathrm{OR}=2.14,95 \% \mathrm{CI}=1.02-4.50)$, while the other subgroup (OTT $\leq 24$ hours) did not show an evident association $(\mathrm{OR}=2.12,95 \% \mathrm{CI}=0.91-4.93)$. Nevertheless, no significant interaction effect was found between OTT $(\leq 24$ or $>24$ hours) and AAR $(>1.53$ or $\leq 1.53)(P=0.56)$. 
Table I The basic characteristics and clinical outcomes in patients with acute ischemic stroke by AAR levels

\begin{tabular}{|c|c|c|c|c|c|}
\hline \multirow[t]{2}{*}{ Basic characteristics } & \multirow{2}{*}{$\frac{Q 1}{n=106}$} & \multirow{2}{*}{$\begin{array}{l}Q 2 \\
n=105\end{array}$} & \multirow{2}{*}{$\begin{array}{l}Q 3 \\
n=107\end{array}$} & \multirow{2}{*}{$\begin{array}{l}Q 4 \\
n=103\end{array}$} & \multirow[t]{2}{*}{$P$-value } \\
\hline & & & & & \\
\hline \multicolumn{6}{|l|}{ Demographic data } \\
\hline Male & $80(75.5)$ & $71(67.6)$ & $57(53.3)$ & $52(50.5)$ & $<0.00$ I \\
\hline Age (years) & $57(50-64)$ & $60(52-69)$ & $65(58-73)$ & $69(63-78)$ & $<0.00$ I \\
\hline \multicolumn{6}{|l|}{ Vascular risk factors } \\
\hline Current smoking & $46(43.4)$ & $43(4 I)$ & $29(27.1)$ & $26(25.2)$ & 0.006 \\
\hline Current alcohol drinking & $24(22.6)$ & $28(26.7)$ & $21(19.6)$ & $\mathrm{II}(10.7)$ & 0.029 \\
\hline Hypertension & $71(67.0)$ & $69(65.7)$ & $74(69.2)$ & $71(68.9)$ & 0.942 \\
\hline Diabetes mellitus & $31(29.2)$ & $30(28.6)$ & $29(27.1)$ & $26(25.2)$ & 0.920 \\
\hline Dyslipidemia & $70(66.0)$ & $63(60.0)$ & $63(58.9)$ & 51 (49.5) & 0.111 \\
\hline \multicolumn{6}{|l|}{ Parameters on admission } \\
\hline Systolic BP (mmHg) & $140(128-160)$ & $142(123-160)$ & $150(133-165)$ & $144(\mid 22-161)$ & 0.153 \\
\hline Diastolic BP (mmHg) & $83(78-92)$ & $82(78-94)$ & $84(78-93)$ & $80(7 \mid-88)$ & 0.013 \\
\hline Baseline GCS & & & & & 0.005 \\
\hline $14-15$ & $93(87.7)$ & 91 (86.7) & $92(86)$ & $72(69.9)$ & \\
\hline $8-13$ & II (10.4) & $9(8.6)$ & $10(9.3)$ & $18(17.5)$ & \\
\hline $0-7$ & $2(1.9)$ & $5(4.8)$ & $5(4.7)$ & $13(12.6)$ & \\
\hline OTT (hours) & $48(12-120)$ & $48(24-96)$ & $24(9-72)$ & $48(20-72)$ & 0.124 \\
\hline \multicolumn{6}{|l|}{ Laboratory test } \\
\hline Fasting glucose (mmol/L) & $5.2(4.4-6.6)$ & $5.1(4.4-7.3)$ & $5.0(4.4-6.4)$ & $5.2(4.5-6.6)$ & 0.938 \\
\hline $\mathrm{TC}(\mathrm{mmol} / \mathrm{L})$ & $4.2(3.6-5.1)$ & $4.2(3.6-4.7)$ & $4.2(3.6-4.8)$ & $4.2(3.5-5.1)$ & 0.968 \\
\hline TGs (mmol/L) & $1.6(1.2-2.2)$ & $1.5(I . I-2.1)$ & $1.4(1.0-2.0)$ & $1.2(0.9-1.6)$ & $<0.00$ I \\
\hline HDL-C (mmol/L) & $1.0(0.9-1.2)$ & $\mathrm{I} .0(0.9-I . I)$ & $1.0(0.9-1.2)$ & $1.1(1.0-1.3)$ & 0.002 \\
\hline LDL-C (mmol/L) & $2.6(2 . I-3.2)$ & $2.6(2.1-3.1)$ & $2.5(2.1-3.0)$ & $2.5(2.1-3.2)$ & 0.873 \\
\hline AST (IU/L) & $20.6(17.3-27.8)$ & $19.5(16.1-23.9)$ & $18.2(|5.3-2|)$ & $19(16-24)$ & 0.003 \\
\hline ALT (IU/L) & $28.8(23.2-40.6)$ & $19.8(16.1-24.2)$ & $14.7(\mid 1.6-17.4)$ & $11.2(8.4-13.7)$ & $<0.00$ I \\
\hline rtPA treatment & $12(11.3)$ & $14(13.3)$ & $15(14.0)$ & $12(11.7)$ & 0.921 \\
\hline TOAST subtype & & & & & 0.007 \\
\hline Large artery atherosclerosis & 45 (42.5) & 34 (32.4) & $37(34.6)$ & $27(26.2)$ & \\
\hline Cardioembolism & $3(2.8)$ & $9(8.6)$ & $7(6.5)$ & $18(17.5)$ & \\
\hline Small-artery occlusion & $47(44.3)$ & $49(46.7)$ & $50(46.7)$ & $54(52.4)$ & \\
\hline Other determined & $3(2.8)$ & $6(5.7)$ & $8(7.5)$ & $3(2.9)$ & \\
\hline Undetermined & $8(7.6)$ & $7(6.6)$ & $5(4.7)$ & $I(I)$ & \\
\hline \multicolumn{6}{|l|}{ Clinical outcomes } \\
\hline mRS score $>2$ at 3 months & $37(34.9)$ & $47(44.8)$ & $46(43.0)$ & $60(58.3)$ & 0.008 \\
\hline NIHSS score $>5$ at admission & $44(4 I .5)$ & $49(46.7)$ & $49(45.8)$ & $59(57.3)$ & 0.132 \\
\hline Bleeding & $4(3.8)$ & $7(6.7)$ & $5(4.7)$ & $5(4.9)$ & 0.806 \\
\hline
\end{tabular}

Notes: Continuous variables are expressed as median (interquartile range); categorical variables are expressed as frequency (percent).

Abbreviations: QI, $\leq$ 0.84; Q2, 0.84-I.I; Q3, I.I-I.45; Q4, >I.45; AAR, aspartate transaminase/alanine transaminase ratio; BP, blood pressure; GCS, Glasgow Coma Scale; OTT, onset to treatment time; TC, total cholesterol; TGs, triglycerides; HDL-C, high-density lipoprotein cholesterol; LDL-C, low-density lipoprotein cholesterol; AST, aspartate transaminase; ALT, alanine transaminase; rtPA, recombinant tissue plasminogen activator; mRS, modified Rankin Scale; NIHSS, the National Institute of Health Stroke Scale.


Figure I Column bar graph of AST, ALT, and AAR levels in good outcome and poor outcome patients.

Notes: The top of the rectangle indicates the median value. A horizontal line above the rectangle indicates the third quartile.

Abbreviations: AST, aspartate transaminase; ALT, alanine transaminase; AAR, aspartate transaminase/alanine transaminase ratio; mRS, modified Rankin Scale. 
Table 2 Adjusted odds ratios and 95\% confidence intervals for clinical outcomes in acute ischemic stroke patients in different levels of AAR

\begin{tabular}{|c|c|c|c|c|}
\hline $\begin{array}{l}\text { Clinical } \\
\text { outcomes }\end{array}$ & QI & Q2 & Q3 & Q4 \\
\hline \multicolumn{5}{|c|}{ Primary outcome } \\
\hline \multicolumn{5}{|c|}{ mRS score $>2$ at 3 months } \\
\hline Model I & I & $1.51(0.86-2.67)$ & $1.38(0.77-2.49)$ & $2.50(1.36-4.60)$ \\
\hline Model 2 & I & $1.55(0.86-2.79)$ & $1.50(0.82-2.74)$ & $2.15(1.14-4.05)$ \\
\hline \multicolumn{5}{|c|}{ Secondary outcome } \\
\hline \multicolumn{5}{|c|}{ NIHSS score $>5$ at admission } \\
\hline Model I & 1 & $1.20(0.68-2.14)$ & $1.05(0.58-1.90)$ & $1.82(0.98-3.35)$ \\
\hline Model 2 & 1 & $1.22(0.68-2.20)$ & $1.10(0.60-2.02)$ & $1.55(0.82-2.92)$ \\
\hline \multicolumn{5}{|l|}{ Bleeding } \\
\hline Model I & I & $1.47(0.33-6.48)$ & $0.60(0.12-3.00)$ & $0.77(0.13-4.74)$ \\
\hline Model 2 & 1 & $1.48(0.33-6.67)$ & $0.67(0.12-3.14)$ & $0.56(0.08-3.79)$ \\
\hline
\end{tabular}

Notes: Model I: adjusted for sex, age, current smoking, alcohol intake, hypertension, diabetes mellitus, dyslipidemia, symptom onset to treatment time, and thrombolytic therapy with recombinant tissue plasminogen activator; Model 2: Model I plus Glasgow Coma Scale.

Abbreviations: Q1, $\leq 0.84$; Q2, 0.84-I.I; Q3, I.I-I.45; Q4, > I.45; AAR, aspartate transaminase/alanine transaminase ratio; mRS, modified Rankin Scale; NIHSS, the National Institute of Health Stroke Scale.

\section{Discussion}

The main finding of this study is that AAR may be a useful predictor of functional outcome at 3 months following the first-ever AIS. This relationship remained even after adjusting for related confounders, including sex, age, current smoking, alcohol intake, hypertension, DM, hyperlipidemia, OTT, GCS score, and rtPA therapy. A value of 1.53 was

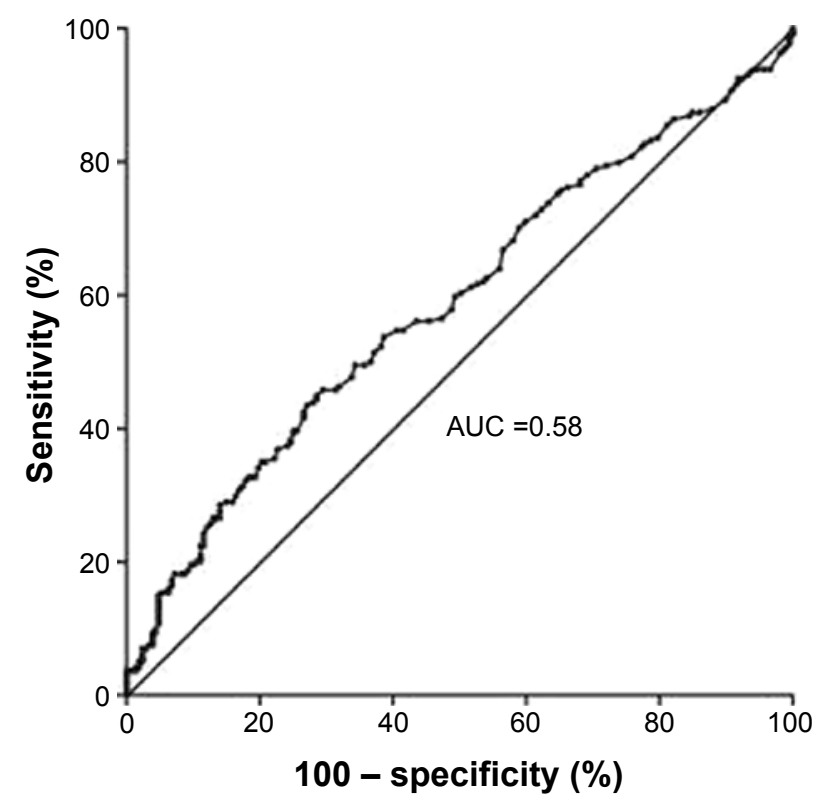

Figure 2 Receiver operating curve of De Ritis ratio for prediction of 3-month poor outcome in acute ischemic stroke.

Notes: AUC: 0.580 (95\% confidence interval 0.525-0.635); $P=0.005$.

Abbreviation: AUC, area under the receiver operating characteristics (ROC) curve.
Table 3 Subgroup analysis of adjusted odds ratios and 95\% confidence intervals of poor outcome according to high AAR (>I.53)

\begin{tabular}{|c|c|c|c|c|}
\hline Subgroup & OR & $95 \% \mathrm{Cl}$ & $P$-value & $P$-interaction \\
\hline Total cohort & 1.89 & $1.11-3.22$ & 0.02 & \\
\hline OTT & & & & 0.56 \\
\hline$\leq 24$ hours & 2.12 & $0.91-4.93$ & 0.08 & \\
\hline$>24$ hours & 2.14 & $1.02-4.50$ & 0.04 & \\
\hline
\end{tabular}

Notes: In the multivariate logistic regression model, sex, age, current smoking alcohol intake, hypertension, diabetes mellitus, dyslipidemia, symptom onset to treatment time, thrombolytic therapy with recombinant tissue plasminogen activator, and Glasgow Coma Scale were included as confounding factors.

Abbreviations: AAR, aspartate transaminase/alanine transaminase ratio; OTT, onset to treatment time; $\mathrm{OR}$, odds ratio; $\mathrm{Cl}$, confidence interval.

found to be the optimal cutoff for continuous AAR, with AAR $>1.53$ at admission being associated with a 1.89-fold greater likelihood of a poor outcome.

The concept of AAR and its correlation with viral hepatitis were first proposed by De Ritis in $1957 .{ }^{23}$ AAR was subsequently used to predict the presence of progressive fibrosis in chronic hepatic B patients and poor survival in chronic viral hepatitis $\mathrm{C} .{ }^{24}$ In recent years, AAR has been shown to be a useful predictor for nonhepatic diseases and a prognostic biomarker in certain types of malignant tumor. ${ }^{11,14-16,24-27}$ For example, in a large European cohort of nonmetastatic renal cell carcinoma patients, Bezan et al identified preoperative AAR as a predictor of a poor clinical outcome. ${ }^{11}$ Nishikawa et al discovered that preoperative AAR was significantly correlated with a fourfold increase in an unfavorable prognosis in patients with upper urinary tract urothelial carcinoma. ${ }^{13}$ Rief et al studied 1,782 consecutive patients with peripheral arterial occlusive disease and reported that AAR at admission was associated with critical limb ischemia. ${ }^{14}$ Tan et al found that AAR might be associated with an increased risk of inferior long-term survival in distal cholangiocarcinoma. ${ }^{27}$ These previous results demonstrate that elevation of AAR increases the risk of adverse events in various diseases, with the thresholds for these elevations being similar to that in the present study.

The prognostic effects of AST and ALT on AIS patients were first reported by Campos et al. Two prospective cohort studies were performed, which showed that lower AST and ALT levels were associated with a poor outcome (mRS score $>2)^{2,3}$ This is consistent with the findings of the present study. Furthermore, Campos et al found the neuroprotective effect of AST in both animal experiments and clinical studies. In their findings, AST activation could induce a reduction of infarct size and smaller edema volume and decrease the rate of sensorimotor deficits in animal models, and both AST and ALT levels in AIS patients were inversely correlated 
with infarct volume, and patients with lower AST tended to experience early neurological deterioration. ${ }^{2,3,28}$ It could be postulated that patients with bigger infarct volume or early neurological deterioration owing to lower AST and ALT levels may have a poorer outcome at 3 months.

The glutamate level in the blood may be responsible for the elevated AST and ALT, which could metabolize glutamate in the peripheral blood and thereby lead to a decline in glutamate. Previous studies have found that excessive glutamate will be released from neurons into the brain parenchyma extracellular space after ischemic stroke. A small increase in the glutamate concentration in the cerebrospinal fluid will cause a pronounced increase in intracellular calcium and trigger neuronal death. Therefore, glutamate has been shown to be related to a greater stroke severity, larger infarct volume, and worse functional outcome..$^{2,3,8,29}$ Under the effect of AST and ALT, the blood glutamate level will decrease and the brain-to-blood glutamate efflux can be accelerated in accordance with the larger glutamate concentration gradient between the brain and blood. ${ }^{3,7}$

The present study contrasts with Campos et al by introducing a novel prognostic biomarker (AAR). The exact mechanism responsible for the relationship between AAR elevation and poor AIS outcome is still obscure and so requires further investigation. It is conceivable that this is due to a difference in activity between AST and ALT. Numerous studies have demonstrated that ALT is primarily enriched in liver tissue, while AST is widely distributed in diverse organs such as the brain, kidney, muscle, and even the heart. This could result in AST remaining in a higher proliferative state than ALT, even when the condition of the patient is deteriorating. ${ }^{11,15,16,24}$ Among patients with a poor outcome, the decrease in the ALT level was larger than that for AST, resulting in a higher AAR being related to a poor outcome.

The present study also investigated the effects of each AAR quartile on stroke prognosis using a multivariate logistic regression model. The harmful effect of the highest AAR quartile on a poor outcome was slightly reduced in Model 2 compared with Model 1. This may indicate that patients with a lower GCS score at admission tend to have a higher AAR.

This study was subject to several limitations. First, it had a retrospective design, and no data on alterations in ALT and AST levels during hospitalization were obtained. The study therefore only investigated the relationship between the parameters at admission and the prognosis. Second, various diseases may affect the serum levels of AST and ALT at admission. Therefore, some other undetected diseases that could alter the AST and ALT concentrations might have been present in this study that could have influenced the predicted effect of AAR on the prognosis of AIS. Finally, some of the patients enrolled in this study had OTTs exceeding 24 hours. Data on the AST and ALT levels at stroke onset might not have been available for these subjects. However, it reveals a real situation where a large proportion of AIS patients in China are admitted to hospital within 7 days after stroke onset. Subgroup analysis in the present study indicated that there was no marked disparity between patients with OTT $\leq 24$ and $>24$ hours. Further exploration in studies with a prospective design and larger sample size is therefore needed to confirm the results reported here.

\section{Conclusion}

An elevated AAR at admission was found to be associated with a poor outcome at 3 months in patients with first-ever AIS. The optimal cutoff for AAR was found to be 1.53 based on ROC curve analysis. The appropriate administration of serum AST and ALT to AIS patients at admission might be an effective therapy for improving their prognosis.

\section{Acknowledgments}

We would like to thank all the members of the Clinical Research Center, the First Affiliated Hospital of Xi'an Jiaotong University for their participation in this study.

\section{Author contributions}

Research idea and study design: FG, J-FH; data acquisition: FG, CC, X-YY, KH; statistical analysis: FG, JL, JZ, X-CM; data interpretation: $\mathrm{CC}, \mathrm{X}-\mathrm{YY}, \mathrm{KH}, \mathrm{J}-\mathrm{FH}$; manuscript drafting: FG, J-FH. All authors contributed toward data analysis, drafting and revising the paper and agree to be accountable for all aspects of the work.

\section{Disclosure}

The authors report no conflicts of interest in this work.

\section{References}

1. Zhao W, An Z, Hong Y, et al. Low total cholesterol level is the independent predictor of poor outcomes in patients with acute ischemic stroke: a hospital-based prospective study. BMC Neurol. 2016;16(1):36.

2. Campos F, Rodriguez-Yanez M, Castellanos M, et al. Blood levels of glutamate oxaloacetate transaminase are more strongly associated with good outcome in acute ischaemic stroke than glutamate pyruvate transaminase levels. Clin Sci (Lond). 2011;121(1):11-17.

3. Campos F, Sobrino T, Ramos-Cabrer P, et al. High blood glutamate oxaloacetate transaminase levels are associated with good functional outcome in acute ischemic stroke. J Cereb Blood Flow Metab. 2011;31(6): 1387-1393.

4. Hawkins RA. The blood-brain barrier and glutamate. Am J Clin Nutr. 2009;90(3):867S-874S. 
5. Gottlieb M, Wang Y, Teichberg VI. Blood-mediated scavenging of cerebrospinal fluid glutamate. J Neurochem. 2003;87(1):119-126.

6. O'Kane RL, Martinez-Lopez I, DeJoseph MR, Vina JR, Hawkins RA. $\mathrm{Na}(+)$-dependent glutamate transporters (EAAT1, EAAT2, and EAAT3) of the blood-brain barrier. A mechanism for glutamate removal. $J$ Biol Chem. 1999;274(45):31891-31895.

7. Teichberg VI, Cohen-Kashi-Malina K, Cooper I, Zlotnik A. Homeostasis of glutamate in brain fluids: an accelerated brain-to-blood efflux of excess glutamate is produced by blood glutamate scavenging and offers protection from neuropathologies. Neuroscience. 2009;158(1): 301-308.

8. Castillo J, Davalos A, Noya M. Progression of ischaemic stroke and excitotoxic aminoacids. Lancet. 1997;349(9045):79-83.

9. Tan G, Lei C, Hao Z, Chen Y, Yuan R, Liu M. Liver function may play an uneven role in haemorrhagic transformation for stroke subtypes after acute ischaemic stroke. Eur J Neurol. 2016;23(3):597-604.

10. Muscari A, Collini A, Fabbri E, et al. Changes of liver enzymes and bilirubin during ischemic stroke: mechanisms and possible significance. BMC Neurol. 2014;14:122.

11. Bezan A, Mrsic E, Krieger D, et al. The preoperative AST/ALT (De Ritis) ratio represents a poor prognostic factor in a cohort of patients with nonmetastatic renal cell carcinoma. J Urol. 2015;194(1):30-35.

12. Fallatah HI, Akbar HO, Fallatah AM. Fibroscan compared to FIB-4, APRI, and AST/ALT ratio for assessment of liver fibrosis in Saudi patients with nonalcoholic fatty liver disease. Hepat Mon. 2016;16(7): e38346.

13. Nishikawa M, Miyake H, Fujisawa M. De Ritis (aspartate transaminase/alanine transaminase) ratio as a significant predictor of recurrence-free survival in patients with upper urinary tract urothelial carcinoma following nephroureterectomy. Urol Oncol. 2016;34(9): 417.e9-417.e15.

14. Rief P, Pichler M, Raggam R, et al. The AST/ALT (De-Ritis) ratio: a novel marker for critical limb ischemia in peripheral arterial occlusive disease patients. Medicine (Baltimore). 2016;95(24):e3843.

15. Lee H, Choi YH, Sung HH, et al. De Ritis ratio (AST/ALT) as a significant prognostic factor in patients with upper tract urothelial cancer treated with surgery. Clin Genitourin Cancer. 2016;S1558-S7673(16): 30257-30259.

16. Lee H, Lee SE, Byun SS, Kim HH, Kwak C, Hong SK. De Ritis ratio (AST/ALT) as a significant prognostic factor after surgical treatment in patients with clear cell localized renal cell carcinoma: a propensity score matched study. BJU Int. 2017;119(2):261-267.
17. Zhong C, Xu T, Xu T, et al. Plasma homocysteine and prognosis of acute ischemic stroke: a gender-specific analysis from CATIS randomized clinical trial. Mol Neurobiol. 2017;54(3):2022-2030.

18. Furlan JC, Fang J, Silver FL. Outcomes after acute ischemic stroke in patients with thrombocytopenia or thrombocytosis. J Neurol Sci. 2016; 362:198-203.

19. Huber T, Kleine JF, Kaesmacher J, et al. Blood leukocytes as prognostic parameter in stroke thrombectomy. Cerebrovasc Dis. 2016;42(1-2): $32-40$.

20. Lei C, Wu B, Liu M, Chen Y. Association between hemoglobin A(1)C levels and clinical outcome in ischemic stroke patients with or without diabetes. J Clin Neurosci. 2015;22(3):498-503.

21. Huang WY, Weng WC, Peng TI, et al. Association of hyponatremia in acute stroke stage with three-year mortality in patients with first-ever ischemic stroke. Cerebrovasc Dis. 2012;34(1):55-62.

22. Fure B, Wyller TB, Thommessen B. TOAST criteria applied in acute ischemic stroke. Acta Neurol Scand. 2005;112(4):254-258.

23. De Ritis F, Coltorti M, Giusti G. An enzymic test for the diagnosis of viral hepatitis: the transaminase serum activities. Clin Chim Acta. 2006;369(2):148-152.

24. Botros M, Sikaris KA. The De Ritis ratio: the test of time. Clin Biochem Rev. 2013;34(3):117-130.

25. Rawson NS, Peto J. An overview of prognostic factors in small cell lung cancer. A report from the Subcommittee for the Management of Lung Cancer of the United Kingdom Coordinating Committee on Cancer Research. Br J Cancer. 1990;61(4):597-604.

26. Stocken DD, Hassan AB, Altman DG, et al. Modelling prognostic factors in advanced pancreatic cancer. Br J Cancer. 2008;99(6):883-893.

27. Tan X, Xiao K, Liu W, et al. Prognostic factors of distal cholangiocarcinoma after curative surgery: a series of 84 cases. Hepatogastroenterology. 2013;60(128):1892-1895.

28. Campos F, Sobrino T, Ramos-Cabrer P, et al. Neuroprotection by glutamate oxaloacetate transaminase in ischemic stroke: an experimental study. J Cereb Blood Flow Metab. 2011;31:1378-1386.

29. Castellanos M, Sobrino T, Pedraza S, et al. High plasma glutamate concentrations are associated with infarct growth in acute ischemic stroke. Neurology. 2008;71(23):1862-1868.
Neuropsychiatric Disease and Treatment

\section{Publish your work in this journal}

Neuropsychiatric Disease and Treatment is an international, peerreviewed journal of clinical therapeutics and pharmacology focusing on concise rapid reporting of clinical or pre-clinical studies on a range of neuropsychiatric and neurological disorders. This journal is indexed on PubMed Central, the 'PsycINFO' database and CAS,

\section{Dovepress}

and is the official journal of The International Neuropsychiatric Association (INA). The manuscript management system is completely online and includes a very quick and fair peer-review system, which is all easy to use. Visit http://www.dovepress.com/testimonials.php to read real quotes from published authors. 